\title{
Estamos olhando para os indivíduos pós-COVID como deveríamos?
}

\author{
Are we looking at post-COVID \\ patients as we should?
}

\section{Christiane Riedi Daniel' ${ }^{1}$ (I) \\ Marina Pegoraro Baroni² (1) João Afonso Ruaro 3 (1) Andersom Ricardo Fréz ${ }^{4}$ (1)}

1-3Universidade Estadual do Centro-Oeste (Guarapuava).Paraná, Brasil. criedi@unicentro.br, marinapegoraro@hotmail.com, joaoruaro@gmail.com ${ }^{4}$ Autor para correspondência. Universidade Estadual do Centro-Oeste (Guarapuava). Paraná, Brasil. andersom_frez@yahoo.com.br

Desde que a pandemia pelo coronavírus se instalou, muito se tem falado e pesquisado a respeito das manifestações clínicas da COVID-19, cujas apresentações variam de assintomáticos, sintomáticos leves, moderados e graves. As principais manifestações são febre, tosse e fadiga podendo evoluir com quadro de pneumonia e síndrome do desconforto respiratório agudo ${ }^{1-3}$.

O padrão de gravidade estudado até o momento subdivide estes indivíduos na seguinte terapêutica: isolamento domiciliar para assintomáticos e sintomáticos leves, internamento nas enfermarias dos hospitais para sintomáticos moderados e suporte ventilatório e cuidados intensivos para sintomáticos graves 4 .

As manifestações clínicas mais estudadas estão voltadas principalmente para a forma mais grave da doença, que envolve internação em unidade de terapia intensiva (UTI), necessidade de suporte ventilatório, altas doses de sedativos, tempo prolongado de hospitalização e alta mortalidade ${ }^{5}$, variando de 50 a $97 \%$ para pacientes que necessitaram de ventilação mecânica ${ }^{6}$. Ainda, $70 \%$ dos indivíduos internados em UTI podem desenvolver diversas outras complicações associadas à internação, as quais impactam no sistema respiratório, cardiovascular, neurológico e musculoesquelético $\stackrel{?}{\text {. }}$

Além da gravidade destes pacientes, tem-se observado que muitas vezes indivíduos acometidos pela COVID-19 apresentam sintomas persistentes mesmo após serem considerados recuperados. Na Itália, um acompanhamento pós-COVID de indivíduos não hospitalizados, demonstrou que $87,4 \%$ dos indivíduos avaliados 60 dias após serem considerados curados ainda apresentavam sintomas persistentes. Destes, 53,1\% indicaram a fadiga como sintoma mais frequente, seguido da dispneia $(43,4 \%)$ e dor articular $(27,3 \%)$, que levaram à uma piora na qualidade de vida em $44,1 \%$ das pessoas $\stackrel{8}{ }$. 
Baseando-se na literatura de reabilitação cardiopulmonar em outras doenças respiratórias ${ }^{9}$, estes sintomas de fadiga e dispneia apresentados pelos indivíduos pós-COVID é indicado ao programa de reabilitação cardiopulmonar precoce pós-COVID ${ }^{10}$. Além disso, para alguns indivíduos também pode ser necessário incluir componentes da reabilitação neuromuscular ${ }^{4}$. Neste contexto, é necessário ampliarmos a visão do cuidado no âmbito da avaliação multidimensional e intervenção para os indivíduos pós-COVID e questionarmos: Os sintomas pós-COVID estão sendo monitorados? O que de fato está sendo feito para minimizar estes sintomas? Quais protocolos estão sendo implementados para estes cuidados? Como estes pacientes estão sendo encaminhados para os serviços de reabilitação?

A reabilitação de pacientes críticos após alta hospitalar de outras doenças já é realizada em todo mundo, justamente para tentar minimizar os efeitos deletérios da internação prolongada, o impacto funcional, econômico, social e laboral a médio e longo prazo ${ }^{z}$. No entanto, orientações sobre a necessidade do manejo em pacientes pós-COVID são escassas na literatura ${ }^{11}$, porém esta discussão se faz necessária e é urgente visto que já se sabe que a COVID-19 resulta em alta incapacidade e morbidade, principalmente nos grupos de risco $^{12}$. No Brasil, a Associação Brasileira de Fisioterapia Cardiorrespiratória e Fisioterapia em Terapia Intensiva (ASSOBRAFIR) auxiliou na capacitação dos fisioterapeutas para atuarem junto aos pacientes acometidos pela COVID-19. Além disso, a ASSOBRAFIR teve a iniciativa de criar o Comitê COVID-19 para produzir documentos norteadores, especialmente as relacionadas à atuação fisioterapêutica ${ }^{13}$.

O monitoramento, avaliação, reabilitação e reavaliação periódica dos indivíduos com pneumonia pós-COVID, síndrome do desconforto respiratório agudo pós-COVID ou aqueles cujos sintomas permanecem mesmo após serem considerados curados da COVID-19 são necessários e imprescindíveis para garantir o retorno pleno do indivíduo às suas funções laborais e sociais com qualidade ${ }^{7,14}$. Mas, para isto, como está sendo realizado este monitoramento pós-COVID? O que tem sido feito para os indivíduos cujos sintomas respiratórios e neuromusculares persistem?

Os governos estão organizados de forma a criar redes de acompanhamento e assistência a estes indivíduos? Os serviços de reabilitação estão estruturados para receber, avaliar, tratar e acompanhar pacientes pós-COVID?
Esta organização para o período pós-pico da pandemia é necessária para minimizar a sobrecarga dos serviços de saúde, os quais já acumulam atendimentos eletivos, e principalmente evitar afastamentos do trabaIho por incapacidades, com impacto econômico ainda maior 11,15 . Iniciativas internacionais para avaliação e acompanhamento das repercussões funcionais apresentadas por pacientes acometidos pela COVID-19 já podem ser identificadas na literatura ${ }^{16}$.

A reabilitação destes indivíduos é primordial e deve ser centrada no paciente e adaptada às suas necessidades ${ }^{14}$, pautada na redução dos sintomas respiratórios, ganho da força, funcionalidade e qualidade de vida $^{4}$. Deve ainda ser constituída por medidas educativas $^{4,11}$ para o entendimento da doença, dos efeitos secundários gerados por ela e para proporcionar condições e estratégias para que o paciente seja protagonista na sua recuperação ${ }^{4}$.

Como a reabilitação deve ser fundamentada na funcionalidade, ações voltadas para atividade e participação social também devem ser consideradas. Para isso, o protocolo de reabilitação deve envolver componentes respiratórios, cardiovasculares, musculoesqueléticos, neurológicos e cognitivo-emocionais ${ }^{12}$ por meio de exercícios de força muscular respiratória e periférica, flexibilidade, exercícios aeróbios, treino de marcha e equilíbrio ${ }^{17}$. Além destas, outras estratégias de reabilitação também podem ser necessárias para alcançar uma menor restrição na participação social, como a reabilitação psicológica ${ }^{4}$. A modalidade da reabilitação pode ser ofertada de diferentes formas, seja pelo atendimento presencial para pacientes com maiores incapacidades, seja por telerreabilitação para indivíduos com menor comprometimento e maior acesso às tecnologias de informação e comunicação (TICS) ou ainda por um modelo híbrido, o qual combine as modalidades ${ }^{18}$.

Neste momento de pandemia, a telerreabilitação tem sido amplamente utilizada e isto não poderia ser diferente com pacientes pós-COVID-19. Sua utilização permite a assistência de um maior número de pessoas mesmo com o número reduzido de profissionais, minimiza a sobrecarga de assistência presencial no serviço público, não necessita aguardar o retorno da assistência eletiva à saúde, extingue o risco de contágio e disseminação da COVID-19, e viabiliza um serviço sem a necessidade do deslocamento dos pacientes permitindo assim uma maior segurança a todos ${ }^{14}$. Porém, ainda existem algumas barreiras em relação ao uso da telereabilitação, como falta de familiaridade dos 
pacientes com as tecnologias e muitas vezes falta de recursos financeiros para adquiri-las. Contudo, esse tipo de reabilitação é promissor e deve ser incentivado por iniciativas governamentais considerando os potenciais benefícios que pode trazer.

Como em todo processo de reabilitação, a adesão ao tratamento é fundamental e as estratégias adotadas pelo profissional impactam diretamente no sucesso da reabilitação. Por isso é necessário que os serviços de reabilitação e os profissionais envolvidos se reestruturem, desenvolvam protocolos e rotinas bem estabelecidas, respeitando a individualidade de cada paciente e pautando-se nas melhores ferramentas de avaliação e intervenção, visando proporcionar um impacto positivo na reabilitação funcional e qualidade de vida dos indivíduos pós-COVID. E que assim possamos refletir: Estamos mesmo olhando para os indivíduos pós-COVID como deveríamos?

\section{Referências}

1. Rothan HA, Byrareddy SN. The epidemiology and pathogenesis of coronavirus disease (COVID-19) outbreak. J Autoimmun. 2020;109:102433. doi: 10.1016/j.jaut.2020.102433

2. Dong X, Cao YY, Lu XX, Zhang JJ, Du H, Yan YQ et al. Eleven faces of coronavirus disease 2019. Allergy. 2020;75(7):1699-1709. doi: 10.1111/all.14289

3. Centers for Disease Control and Prevention. Coronavirus disease 2019 basics. [Internet]. 2020. [accessed 2020 set 29]. Available from: https://www.cdc.gov/coronavirus/2019-ncov/faq. html\#Coronavirus-Disease-2019-Basics.

4. Barker-Davies RM, O'Sullivan O, Senaratne KPP, Baker P, Cranley M, Dharm-Datta S et al. The Stanford Hall consensus statement for post-COVID-19 rehabilitation. Br J Sports Med. 2020;54(16):1-11. doi: 10.1136/bjsports-2020-102596

5. Bajwah S, Wilcock A, Towers R, Constantini M, Bausewein C, Simon ST al. Managing the supportive care needs of those affected by COVID-19. Eur Respir J. 2020;56(4). doi: 10.1183/13993003.00815-2020

6. Auld SC, Caridi-Scheible M, Blum JM, Robichaux C, Kraft C, Jacob JT et al. ICU and Ventilator Mortality Among Critically III Adults With Coronavirus Disease 2019. Crit Care Med. 2020;48(9):e799-e804. doi: 10.1097/CCM.0000000000004457

7. Smith JM, Lee AC, Zeleznik Het, Scott JPC, Fatima A, Needham $\mathrm{DM}$ et al. Home and community-based physical therapist management of adults with post-intensive care syndrome. Phys Ther. 2020;100(7):1062-1073. doi: 10.1093/ptj/pzaa059
8. Carfi A, Bernabei R, Landi F. Persistent Symptoms in Patients After Acute COVID-19. Jama. 2020;324(6):603-605. doi: 10.1001/ jama.2020.12603

9. McCarthy B, Casey D, Devane D, Murphy K, Murphy E, Lacasse Y. Pulmonary rehabilitation for chronic obstructive pulmonary disease. Cochrane Database Syst Rev. 2015;23(2):Cd003793. doi: 10.1002/14651858.CD003793.pub3

10. Grigoletto I, Cavalheri V, Lima FF, Ramos EMC. Recovery after COVID-19: The potential role of pulmonary rehabilitation. Braz J Phys Ther. 2020. doi: 10.1016/j.bjpt.2020.07.002

11. Stam HJ, Stucki G, Bickenbach J. Covid-19 and post intensive care syndrome: a call for action. J Rehabil Med. 2020;52(4):jrm00044. doi: 10.2340/16501977-2677

12. Candan SA, Elibol N, Abdullahi A. Consideration of prevention and management of long-term consequences of post-acute respiratory distress syndrome in patients with COVID-19. Physiother Theory Pract. 2020;36(6):663-668. doi: $\underline{10.1080 / 09593985.2020 .1766181}$

13. Matte DL, Mesquita R, Furlanetto KC, Karsten M. ASSOBRAFIR reforça sua missão no enfrentamento à pandemia da COVID-19. ASSOBRAFIR Ciência. 2020;11(Supl 1):11-13. doi: 10.47066/21779333.AC20.covid19.001

14. Gonzalez-Gerez JJ, Bernal-Utrera C, Anarte-Lazo E, Garcia-Vidal JA, Botella-Rico JM, Rodriguez-Blanco C. Therapeutic pulmonary telerehabilitation protocol for patients affected by COVID-19, confined to their homes: study protocol for a randomized controlled trial. Trials. 2020;21:588. doi: 10.1186/s13063-02004494-w

15. Polastri M, Nava S, Clini E, Vitacca M, Gosselink R. COVID-19 and pulmonary rehabilitation: preparing for phase three. Eur Respir J. 2020;55(6):2001822. doi: 10.1183/13993003.01822-2020

16. Klok FA, Boon GJAM, Barco Set al, Geelhoed M, Knauss S, Rezek SA et al. The Post-COVID-19 Functional Status scale: a tool to measure functional status over time after COVID-19. Eur Respir J. 2020;56(1):2001494. doi: 10.1183/13993003.01494-2020

17. Chinese Association of Rehabilitation Medicine; Respiratory Rehabilitation Committee of Chinese Association of Rehabilitation Medicine; Cardiopulmonary Rehabilitation Group of Chinese Society of Physical Medicine and Rehabilitation. [Recommendations for respiratory rehabilitation of coronavirus disease 2019 in adult]. Zhonghua Jie He He Hu Xi Za Zhi. 2020;43(4):308-314. doi: 10.3760/cma.j.cn112147-20200228-00206

18. Cacau LAP, Mesquita R, Furlanetto KC, Borges DLS, Forgiarini Junior LA, Maldaner $V$ et al. Avaliação e intervenção para a reabilitação cardiopulmonar de pacientes recuperados da COVID-19. ASSOBRAFIR Ciência. 2020;11(Supl 1):183-193. doi: 10.47066/2177-9333.AC20.covid19.018 